\title{
REPORTS"OF THE MEDICAL SOCIETIES.
}

Edinburgh Medico-Chirurgical Societr, December 4, 1901.Professor T. R. Fraser, President, in the chair.-Drs. W. Elder and Miles showed a patient after removal of a tumour from the left prefrontal lobe of the brain.-Drs. Lovell Gulland and Logan Turner showed a patient after operative treatment for laryngeal tuberculosis.Mr. Shaw M'Laren showed a patient after recovery from compound depressed fracture of the skull and lepto-meningitis. - Dr. Wr. ELder and Mr. Miles showed a tumour removed from left prefrontal lobe of the brain.-Dr. F. D. Boyd showed the bowel from a case of acute dysentery.-Dr. Burn Murdoch showed large gall-stones which produced practically no symptoms.-Mr. Alexis Thomson showed a large gall-stone which caused acute intestinal obstruction, and was removed by enterotomy. - Dr. Harver Litrlejohn showed-(1) Surgical instruments used by natives of Northern Nigeria. Kidney and ureter, showing effect of accidental ligature of ureter for removal of intra-ligamentous ovarian cyst. (3) Injuries received in a fall from a window twenty-five feet high.- Mr. W. Cathcart showed-(1) A toy whistle removed from the trachea of a boy, æt. 9, after being six weeks in the air-passages. (2) Portions of needle and thread, also Röntgen-ray photograph of same, removed from the knee of a girl, æt. 7.-Mr. CAIRD showed-(1) Carcinoma of ascending colon, from a case of primary resection and suture. (2) Lung preparations (method of Buchhold).-Dr D. C. WATson showed photographs of poultry, showing some results of an experimental research on the comparative pathology of gout.-Mr. DAvin W ALlace showed Beckmann's apparatus for ascertaining the freezing-point of blood.-Dr. DAwson TURNER-(1) Apparatus for measuring the electrical resistance of blood. (2) Note on the Röntgen rays in syphilis.-Dr. Lawson of Nordrach-onDee read a paper on "Some Clinical Observations upon the Temperature of Phthisis," based upon experience of one hundred consecutive cases treated by open-air methods. Dr. Lawson first described his method of recording temperature, pulse-rate, and weight. He`took the highest and lowest points of every four consecutive days and averaged them. The average charts thus obtained gave the amplitude, which decreases in favourable and increases in unfavourable cases. The temperature and weight should improve together. In unfavourable cases a patient may gain weight and yet be rapidly getting worse. The diazo reaction in the urine is a very unfavourable sign. The temperature of phthisis presents two features-inelasticity and instability. By inelasticity is meant the fact that the temperature does not readily fall after the exciting cause is removed, e.g. the hill-climbing test. By 
instability is meant that the temperature is easily raised by slight causes, such as an attack of indigestion, variation in temperature of the atmosphere, and excitement. Excessive heat of the air may cause a very considerable rise. In the taking of temperature Dr. Lawson strongly advocated the rectal method, because (1) external influences are not important; (2) the temperature is higher, absolutely and relatively; and (3) because it is more sensitive. The temperature is the best guide as to the effect of treatment, and should be taken regularly and frequently. As a rule, the temperature is highest at 2 a.m., and lowest in the evening. An inverse temperature range is a very bad type. Faulty temperature records are responsible for many errors in diagnosis. Exercise in a healthy individual may raise the temperature, but it quickly falls again to normal ; in the tuberculous subject exercise may cause a fall, - as, for example, in fibroid phthisis, the fall being due to exhaustion,- - or it may cause a rise, and this rise takes a longer time to fall than in the healthy. In a favourable case the temperature should fall again in an hour. In considering a case, an all-round view of it should be taken, the temperature, pulse, weight, and condition of the lungs being all fully considered.-Mr. D. WALLACE read a paper on "Suprapubic Prostatectomy, with Notes on Eleven Cases." This operation is said to be a dangerous one per se, but Mr. Wallace has not found it so. The mortality of castration and vasectomy is as high as that of prostatectomy. Suprapubic operation is much better than the perineal, because inspection is easier and the size of the prostate does not interfere, and further procedure can be adopted. It is impossible to remove the prostate without opening the urethra. Prostatic hypertrophy is of three kinds - vascular, fibrotic, and fibro-adenomatous. Catheter life should be initiated before atony of the bladder sets in: if there be much difficulty in using the catheter, castration should be performed. In later cases where atony has set in, or where there are complications, the suprapubic operation should be performed. Bleeding is easily arrested by washing out freely. Sprengel's pump, as modified by Mr. Cathcart, is the best method of draining the bladder. Of eleven cases operated on, five were cured, three improved, one relapsed in statu $q u o$, two were done quite recently, so it is too early to pronounce on them.

Edinburgh Medico - Chirurgical Society. - Clinical Meeting, Derember 18, 1901.-Dr. Andrew in the chair.-Mr. Cathcart showed a patient after drainage of both pleural cavities for double empyema.-Dr. W. Allan Jamieson showed-(1) A case of lupus vulgaris treated by means of the Finsen light treatment, and apparently cured; $(2)$ case of rodent ulcer treated by X-rays.-Mr. NiLes showed-(1) A patient after operation (posterior gastro-enterostomy) performed for severe hæmatemesis due to a gastric ulcer situated close to the pylorus; (2) a patient after operation for intestinal obstruction due to tuberculous peritonitis, subsequent development of tuberculous pleurisy, which was treated by drainage (operations August and October 1898); (3) patient after excision of the rectum by Kraské's method.-Dr. W. G. Sym showed a patient on whom double excision of the lachrymal sac was performed, followed by extraction of cataract in one eye--Mr. STILES showed-(1) A boy after 
operation for recurrent tubercle and stricture following amputation of the penis for tuberculous disease of the glands ; (2) child, æt. 14 months, after operation for congenital malformation of the anus; (3) several patients, showing the results of treatment of congenital wry-neck by the "open operation," which consists in exposing the muscle and dividing it and the subjacent fascia.-Mr. Alexis Thomson showed a female patient, æt. 52 years, the pyloric portion of her stomach having been removed for cancer three and a half years ago.-Mr. CAIRD showed a patient after operation for high shoulder, due to an osseous plate developed between the scapulæ and vertebral spines.-Mr. MiLes showed(1) Rectum excised for malignant disease by Kraské's operation ; (2) specimens of præputial calculi; (3) specimens illustrating fracture dislocation of the spine.-Mr. CAIRD showed-(1) Specimens and photographs from case operated on for high shoulder; (2) carcinoma of transverse colon, from a case of resection and primary suture.

Edinburgh Obstetrical Society.-December 11, 1901.-Dr. James Ritchie, President, in the chair.-Dr. Berry Hart showed-(a) A large fibroid which had burrowed between the layers of the meso-rectum; (b) two tuberculous kidneys, one removed by abdominal section, the other by lumbar nephrectomy; $(c)$ an ovarian tumour with twisted pedicle.-Dr. Milne Murray showed-(a) Two early chorionic sacs; (b) the entire gestation sac from a case of placenta prævia at the seventh month.-Dr. Lamond Lackie showed an ovarian tumour with a twisted pedicle.-Dr. Haultain showed the uterus from a case of hæmatometra.

Communications :-Dr. Milne Murray read a paper on "A Case of Spontaneous Rupture of an apparently Normal Uterus at the Commencement of Labour." He first of all referred to the various conditions under which rupture of the uterus occurs. He then described a case in which none of the ordinary conditions supposed to favour rupture seemed to be present. When Dr. Murray saw the patient he found her in a condition of collapse, and on examination under chloroform he determined that the child was in the abdominal cavity, and per vaginam an extensive rent in the uterus wall could be detected. Though the chances of recovery were remote, Dr. Murray decided that abdominal hysterectomy was the only possible means of saving the woman's life, and this operation he performed as speedily as possible. There were many difficulties; but except for a pelvic abscess, which for a time complicated the convalescence, the patient did well, and at the present moment was enjoying good health.-2. Dr. Munro Kerr (Glasgow) read a paper on "The Separation of the Placenta, with special reference to the information derived regarding the Mechanism from cases of Conservative Cæsarean Section." He first pointed out the limitations to the deductions drawn from clinical observations of normal labours and from frozen sections regarding the mechanism of the third stage of labour. Dr. Kerr then gave details of what he had observed with regard to the separation of the placenta in eight cases of Cæasarean section which he had performed this year.

Glasgow Medico-Chirurgical Society, November 15, 1901.-Dr. Middeton and Dr. M'VAIL introduced a discussion on "The Treat- 
ment of Pneumonia." Dr. Middleton discussed the etiology and symptoms of pneumonia. He was inclined to regard it as a specific febrile disorder. As regards treatment, he had nothing new to add. The treatment must be that of febrile disorder, namely, the maintenance of the patient's strength. Local applications were useful in the early stages. Stimulants, he thought, were given to too great an extent and in too large doses. To brace up the heart, strychnine was more useful than digitalis; it had to be given hypodermically. For sleeplessness and delirium he gave opium and morphia in the early stages; in the later stages he thought they interfered with the respiration. $\mathrm{He}$ had also found useful for these symptoms chloral and bromide, paraldehyde and hyoscine. He was not impressed with the benefits derived from the administration of oxygen. Dr. M'Vail mentioned two cases where the gravity of the illness fell upon the spleen. One came to post-mortem: the spleen was found loaded with pneumococci. He mentioned also the case of a boy who was admitted to hospital with a resolving pneumonia. On the forehead there was an erysipelatous blush, which spread over the face and head. Over the face large bullæ formed, the fluid from which showed abundant pneumococci. As regards treatment, he gave milk, and milk only. Alcohol he gave as a food and not as a stimulant; he gave it liberally. Oxygen he had found very valuable. He thought it should be administered freely. He had given as much as 1000 cubic feet of oxygen in ten days. For sleeplessness he gave opium in all stages, unless there was bronchitis. He did not believe in expectorants, as nearly all the material from the lung was absorbed and very little expectorated. Dr. M'Call Anderson considered the best treatment to be food and careful nursing. For pain and sleeplessness he gave morphia in all stages, unless there was œdema and bronchitis. Delirium he considered to be often the result of high fever, and to be best treated in such cases by antipyretics. For hyperpyrexia and prolonged pyrexia he applied ice-bags to the head and affected side. If this was not successful he gave quinine, and as a last resource he used the cold bath. He thought most cases were the better of a little alcohol, especially in the later stages. For heartfailure he gave liquor strychnina hypodermically, or $\frac{1}{30} \mathrm{gr}$. of digitalis. He had not seen much benefit from the use of oxygen. Dr. Stockman discussed treatment fully. He recommended that the diet should not consist exclusively of milk. He always prescribed m.s. of tr. strophanthi several times daily from the outset. Alcohol he did not give until there were signs of heart-failure, as the effect could be got at once. He did not consider it of much value as a food. Expectorants in pneumonia were more useful for their effect on the heart than on the lungs. Strychnine was not, in his opinion, equal to alcohol as a cardiac stimulant. The only contra-indications to morphia were bronchitis and meningitis. He had not seen much benefit from oxygen.

Glasgow Pathological and Chinical Society, December 9, 1901.The President, Mr. Henry Clark, in the chair.-Dr. Finlayson gave a demonstration of works on pathological anatomy, principally from the library of the Faculty of Physicians and Surgeons of Glasgow, of which he was for many years the honorary librarian. After a brief sketch of the 
history of the subject, Dr. Finlayson commenced the demonstration with the first work specially devoted to pathology-the "Sepulchretum" of Bonetus, published in 1679. He then traced the progress of the science by the great work of Morgagni, "De Sedibus et Causis Morborum," the museums of William and John Hunter, Natthew Baillie's "Morbid Anatomy," and the works of other pioneers, up to the great wealth of writings and illustration of the latter half of last century. A most interesting demonstration, which was highly appreciated by the members of the Society.

Glasgow Medico-Chirurgical Society. - December 20, 1901.Dr. Archibald Young showed a simple form of gauge for investigation of spinal and other variations of symmetry.-Dr. T. K. Monno showed a young woman with enlarged cirrhotic liver, enlarged spleen, and albuminuria. The patient, a female, æt. 22 , was admitted to hospital in October 1901, with the history of having been ill for a year with fulness about the waist and swelling of the legs. The liver was enlarged; its surface and lower margin were roughened with nodules. The spleen was two inches below the costal margin; it was tender, smooth, and firm. Heart and lungs were normal. The urine contained much albumin and hyaline and granular casts. Dr. Monro was of opinion that the enlargement of the liver was due to syphilis, and that the enlargement of the spleen and the albuminuria were due to amyloid disease.-Dr. T. K. Monro described for himself and Dr. J. A. Campbell a case which presented the clinical and post-mortem phenomena of enteric fever, but in which Eberth's bacillus was not found, and in which a general infection by staphylococcus pyogenes aureus existed. The patient, a sailor, æt. 21, had been ill for a fortnight before admission, and had been performing his ordinary duties all this time. He died suddenly five days after admission. Postmortem the usual lesions were found in the small intestine, and the spleen and mesenteric glands were enlarged. - Dr. Lindsay Steven showed blood films from a fatal case of broncho-pneumonia in a child æt. 22 months, showing a marked leucocytosis and changes in the staining reaction of the polymorphonuclear leucocytes. The white blood corpuscles numbered 236,000 per cubic mm., the red blood corpuscles 4,800,000 per cubic mm., and the hæmoglobin was 90 per cent. The polymorphonuclear leucocytes showed eosinophile staining and eosinophile granules.

Forfarshime Medical Association, December 5, 1901.-Professor M'Ewan, President, in the chair.-Professor Sutherland sent a number of specimens for exhibition at the meeting.-Professor Ky $\mathrm{xocH}$ read a paper on six cases of abdominal section for pelvic affections presenting points of interest-(1) Case of repeated ectopic pregnancy in the same patient. (2) Repeated ovariotomy; the first operation complicated with pregnancy at fourth month. (3) Repeated ovariotomy within two years. (4) Repeated ovariotomy; the first operation complicated with pleural effusion. (5) Ovariotomy, illustrating the condition of pseudomyxoma of peritoneum. (6) Ovariotomy, complicated with scarlatiniform rash.Dr. Halley read a paper on the "Surgical Treatment of Sciatica." In 
it he restricted himself to nerve-stretching. He referred briefly to its first introduction into this country, and said he did not consider it was now done often enough, giving some hospital statistics to support his view. He quoted the views of various authors on the effect and results of nerve-stretching. He considered it was a very effectual form of counter-irritation; pointed out the advisability of traction on proximal and distal ends of the nerve, and of separating the adhesions as far as possible. Bloodless nerve-stretching was considered harmful without an anæsthetic. The notes of one case were given in support of this. Notes of six cases of the open method of stretching the sciatic nerve were given, in all of which the pain had been relieved, and had remained away in one after it had existed for five years, and in another for eight years. In one of the cases, paralysis of extensors of the ankle and peronei had followed, and continued for three months, but then seemed to be passing off. He concluded by expressing the view that nervestretching of the sciatic should be done earlier, and if pains did recur, nerve-stretching should then be performed on the branches.

\section{OBITUARY.}

\section{Archibald Campbell Clark, M.D., F.F.P.S.G.}

AFTEr a long and painful illness, during which he was almost entirely set aside from active work, Dr. Clark died on the 28th November last. A man of wide attainments and strong individuality, occupying a prominent place in the profession, he naturally was regarded with esteem by the members of the specialty to which he belonged. To an almost unrivalled knowledge of the management and working details of lunatic asylums he added, what is by no means a commonly associated quality, a literary and clinical knowledge of mental diseases and their treatment. For many years he lectured on insanity in St. Mungo's College, Glasgow ; and in 1897 he published a text-book on mental diseases, which, although not meeting with that success which is commutable into common gold, is yet generally admitted to contain, in respect to many subjects,-e.g. puerperal mental affections,-contributions equal to any in the English language.

After a distinguished course of study at the University of Edinburgh, where he graduated in 1878 , Dr. Clark almost immediately began asylum work by becoming assistant to the late Dr. Grierson at the Roxburgh District Asylum, Melrose. From there he passed to Morningside, where he completed his training under Dr. Clouston. In 1880 he was appointed Medical Superintendent of the Kirklands Asylum, Bothwell. His stay there will always be associated with the training of attendants -in which work he was a pioneer-and with the conjoint compilation of a handbook on the nursing of the insane, which has passed through several editions, and is the recognised manual of the Medico-Psycho- 